\title{
Evaluating Overall Survival and Competing Risks of Survival in Patients With Early Stage Breast Cancer Using a Comprehensive Nomogram
}

\section{Yanbo Xu}

the Second Affiliated Hoaptal of Zhejiang University School of Medicine https://orcid.org/0000-00034129-7376

\section{Hong Liu}

the Second Affiliated Hospital of Zhejiang University School of Medicine

\section{Qi-Hua Cao}

the Second Affiliated Hospital of Zhejiang University School of Medicine

Jia-Li Ji

Affiliated Cancer Hopital of Nantong University

\section{Rong-Rong Dong}

the Children's Hospital of Zhejiang University School of Medicine

Dong Xu ( $\nabla$ xudongzju@zju.edu.cn )

\section{Research article}

Keywords: breast cancer, overall survival, cancer-specific survival, nomogram, prognosis prediction

Posted Date: September 24th, 2019

DOI: https://doi.org/10.21203/rs.2.14758/v1

License: (c) (i) This work is licensed under a Creative Commons Attribution 4.0 International License. Read Full License

Version of Record: A version of this preprint was published at Cancer Medicine on April 20th, 2020. See the published version at https://doi.org/10.1002/cam4.3030. 


\section{Abstract}

BackgroundPatients with early stage breast cancer $(\mathrm{BC})$ live long but accompany with competing comorbidities. This study aims to estimate the impact of cancer and non-cancer causes of death in early stage BC patients, and further quantify the survival differences. MethodsPatients diagnosed with breast cancer between 2010 and 2016 from the Surveillance, Epidemiology, and End Results database were enrolled in the study. Cumulative incidence function (CIF) for cause-specific death and other causes of death were estimated, and the differences were tested by Gray's test. Nomogram for estimating 3-, 4-, and 5-year overall survival, cancer-specific survival and other-cause-specific survival was established based on Cox regression analysis and Fine and Gray's competing risk analysis. The discriminative ability, calibration and precision of the nomogram was evaluated and compared using $\mathrm{C}$ statistics, calibration plots and the area under receiver operating characteristic curve. Results196304 eligible patients with early-stage BC patients were enrolled in this study. Prolonged overall survival (OS) was associated with younger age, well differentiation, smaller tumor size, Luminal subtype and presence of surgery ( $p$ $<0.001$ ). For competing events, Fine and Gray's competing risk analysis was used to validate the predictors: i ncreasing age, poorer differentiation, larger tumor size, triple negative subtype, HER2 enriched subtype and absence of surgery for cancer-specific mortality (CSM); and increasing age, larger tumor size and absence of surgery for other-cause-specific mortality (OCSM). The established nomogram was well calibrated, and displayed good discrimination in both training cohort and validation cohort by calibration plots (Figure 4), with a concordance index of 0.801 (95\% Cl, 0.795-0.806; $p=0.003$ ) for OS prediction; $0.830(95 \% \mathrm{Cl}, 0.824-0.836 ; p=0.003)$ for CSM prediction and $0.806(95 \% \mathrm{Cl}, 0.798-0.814 ; \mathrm{p}=0.004)$ for OCSM prediction. Furthermore, the AUC values for predicting survival and death were: OS, $80.2 \%$ (3-year), 79.5\% (4-year), and 78.7\% (5-year); CSM, 83.0\% (3-year), 81.7\% (4-year), and 80.3\% (5-year); OCSM 81.3\% (3-year), 80.8\% (4-year), and 81.7\% (5-year) (Figure 5). ConclusionsWe evaluated OS and CIF of cancerspecific death and other-cause-specific death in patients with early stage BC based on Cox regression analysis and Fine and Gray's competing risk analysis and developed the first comprehensive prognostic nomogram.

\section{Background}

Breast cancer (BC) is the most common malignant tumor among women and the main cause of cancerspecific death, with 268600 estimated new cases and 41700 estimated deaths in 2019 in the USA [1]. Nowadays, the prognosis of $\mathrm{BC}$, especially early stage $\mathrm{BC}$, has been improved dramatically by multidisciplinary treatments including radical resection, neo-/adjuvant chemotherapy, hormone and targeted therapy [2-4]. Moreover, in developed countries, early stage BC has become the most frequently diagnosed invasive breast diseases. However, $\mathrm{BC}$ survivors face several comorbidities as age increases, such as heart and cerebrovascular disease, which increase their risk of death from competing causes other than primary breast cancer. So taking the competing risks into consideration is necessary when assessing the prognosis. Many published studies [5-9] have reported the prognosis of BC, but most of them either just paid more attention on overall survival (OS) or analyzed cancer-specific mortality using traditional Cox regression model which can not necessarily reflect the effect on cumulative incidence. In 
the individualized treatment era, evaluating the OS is not far enough. It is important to differentiate cancer-specific and non-cancer-specific death. When competing risks exist, traditional Cox regression model may be inappropriate, because in this model, the competing events are regarded as censoring and the cancer-specific mortalities may be overestimated [10-12]. So in this situation, the Fine and Gray model $[11,13]$ which takes into account the censoring is recommended. Therefore, we performed a retrospective competing risk analysis and built a comprehensive nomogram to fully evaluate the OS, cancer-specific survival (CSS) and other-cause-specific survival (OCSS) in a large population of early stage BC.

\section{Methods}

\section{Patients}

Data of patients with early stage (stage I-II) breast cancer were retrospectively extracted from the SEER database (2010-2016), using the SEER*Stat version 8.3.4. Totally, we identified 446806 patients who were pathologically diagnosed as breast cancer. The exclusion criteria was as follow: (1) patients with stage IIIIV breast cancer ( $\mathrm{N}=90673)$; (2) patients combined with other primary cancer(s) (N=95070); (3) patients diagnosed at autopsy ( $\mathrm{N}=434)$; (4) patients with censored or incomplete information about survival time $(N=6)$, survival status $(N=244)$, primary tumor size $(N=36927)$, subtype $(N=12347)$, primary site surgery $(\mathrm{N}=190)$ and grade $(\mathrm{N}=6233)$; (5) patients with survival time less than one month ( $\mathrm{N}=8806)$; (6) patients younger than 18 years $(\mathrm{N}=6)$; finally, leaving 196304 patients in the final cohort for analysis. The detailed patient selection criteria was shown in Figure 1. Informed consent was not required because the SEER database contained no personal information. Clinicopathological variables selection was depended on clinical importance and predictors identified in previous studies $[6,8,9]$, including age, grade, tumor size, subtype, surgery to primary sites and survival time. We classified age at diagnosis into seven groups: $<60$ years, $60-65$ years, 66-70 years, 71-75 years, $76-80$ years, $81-85$ years and $>85$ years. The tumor size classifications were categorized into five groups $(<1 \mathrm{~cm},<2 \mathrm{~cm},<3 \mathrm{~cm},<4 \mathrm{~cm}$ and $\geq 4 \mathrm{~cm})$ for OS analysis, six groups ( $<1 \mathrm{~cm},<2 \mathrm{~cm},<3 \mathrm{~cm},<4 \mathrm{~cm},<5 \mathrm{~cm}$ and $\geq 5 \mathrm{~cm}$ ) for cancer-specific mortality (CSM) analysis and four groups $(<1 \mathrm{~cm},<2 \mathrm{~cm},<3 \mathrm{~cm}$ and $\geq 3 \mathrm{~cm}$ ) for other-cause-specific mortality (OCSM) analysis.

Subsequently, the 196304 stage $\mathrm{I}-\mathrm{Il}$ breast cancer patients were then randomly divided into two groups at a ratio of nine to one: training cohort $(\mathrm{N}=176671)$ and validation cohort $(\mathrm{N}=19630)$. Training cohort was used to construct nomogram and validation cohort was served as validation. There was no significant differences between the two groups ( $p>0.05)$ (Table 1$)$.

\section{Statistical analysis}

Demographic and clinical characteristics were summarized using descriptive statistics. Categorical variables were reported as whole numbers and proportions, and continuous variables were reported as medians with interquartile ranges (IQRs) unless indicated otherwise. Chi-square test and Fisher's exact test for categorical variables and Student's $t$ test for continuous variables were calculated to compare baseline characteristics. OS was defined as the time from diagnosis to any cause of death. The Kaplan- 
Meier method was used to generate OS, and the log-rank test was used to exam the differences in OS. CSM and OCSM were termed as two competing events. The associations of relevant clinical variables with OS, CSM, and OCSM were analyzed using Cox regression model and the Fine and Gray's competing risk analysis (based on the subdistribution hazard ratio) $[11,13,14]$. The CSM and OCSM were presented by cumulative incidence function (CIF) and the differences were assessed using Gray's test. The independent risk factors identified in the multivariate analysis were incorporated into the nomogram to predict the probability of 3-, 4-, and 5-year OS, CSS and OCSS rates for patients with early stage BC using the rms and mstate package in $\mathrm{R}$ project $[10,15]$. The ability and calibration of the nomogram was assessed by concordance index (C-index) and calibration curves (comparing the nomogram-predicted probability with the observed probability) $[16,17]$. Besides, the calibration curves were used to reduce the overfit bias via a bootstrap method with 1000 resamples. Furthermore, the precision of the 3-, 4-, and 5year OS, CSM and OCSM of the nomogram was evaluated and compared by using AUC. Larger C-index and AUC value show higher ability to distinguish patients from different survival outcomes. Last, KaplanMeier curves were plotted over patients grouped by risks predicted from the nomogram to further assess calibration [18]. A two tailed $p<0.05$ was considered statistically significant. All analyses were conducted using R (version 3.4.3; R Foundation).

\section{Results}

\section{Patient}

196304 early stage BC patients from 2010 to 2016 were obtained for the final analysis and divided into two groups at a ratio of nine to one: training cohort $(\mathrm{N}=176671)$ and validation cohort $(\mathrm{N}=19630)$. The baseline characteristics of the two groups were summarized in Table 1 and there was no significant difference between them ( $p>0.05)$. The median age at diagnosis was 60 years (IQRs, 51-70 years). Among the whole population, nearly a half of the patients (47.5\%) were younger than 60 years old. Moderate differentiation (Grade II) (44.4\%) accounted for the highest proportion, followed by poor differentiation (Grade III-IV) (30.3\%), and well differentiation (Grade I) (25.3\%). Small tumors were prevailing in earlystage BC patients. $62.7 \%$ of the tumor sizes were smaller than 2 centimeter $(\mathrm{cm})$ and only $2.8 \%$ of tumors were larger than $5 \mathrm{~cm}$. Most patients $(75.1 \%)$ were categorized as Luminal A subtype (hormone receptor [HR]+/ human Epidermal growth factor receptor-2 [HER2]-), followed by Triple Negative subtype (HR-/HER2-) (10.8\%), Luminal B subtype (HR+/HER2+) (10.2\%) and HER2 Enriched subtype (HR-/HER2+) $(3.9 \%)$.

\section{Survival}

The median follow-up time was 41 months (IQRs, 24-60 months). 12417/196304 (6.3\%) patients died: $5628 / 12417(45.3 \%)$ as a result of cancer-specific deaths and $6789 / 12417(54.7 \%)$ as a result of other- 
cause-specific deaths. The 3-, 4-, and 5-year overall survival rates, cancer-specific mortalities and othercause-specific mortalities which were stratified by age, grade, tumor size, subtype and surgery were listed in Table 2.

On multivariable Cox regression (Table 3), older age ( $p<0.001)$, poorer differentiation (Grade II vs. Grade l; hazard ratio [HR], 1.135; 95\% confidence interval [Cl], 1.075-1.199; $p<0.001$; Grade III-IV vs. Grade l; HR, $1.703 ; 95 \% \mathrm{Cl}, 1.602-1.809 ; p<0.001)$, larger tumor size ( $p<0.001)$, triple negative subtype (vs. luminal $\mathrm{B}$ subtype; HR, 1.859; 95\% Cl, 1.724-2.004; $p<0.001$ ), HER2 enriched subtype (vs. luminal B subtype; HR, $1.167 ; 95 \% \mathrm{Cl}, 1.048-1.300 ; p=0.005$ ), and absence of surgery (vs. surgery; $\mathrm{HR}, 3.428 ; 95 \% \mathrm{Cl}, 3.277-3.641$; $p<0.001$ ) were significantly associated with a poorer OS. For CSM and OCSM, Fine and Gray's competing risk analysis was used and the following factors were validated (Table 3): older age, poorer differentiation, larger tumor size, triple negative subtype, HER2 enriched subtype and absence of surgery for CSM; and older age, larger tumor size and absence of surgery for OCSM. Age was a strong predictive factor and more obvious on OCSM $(p<0.001)$. The OCSM were significantly elevated in patients with age increasing: compared with the young patients, the elderly patients carried higher competing risks (60-65 years vs. $<60$ years; subdistribution hazard ratio [SHR], 2.561, 95\% Cl, 2.283-2.873; $p<0.001 ; 66-70$ years vs. $<60$ years; $S H R, 4.182,95 \% \mathrm{Cl}, 3.742-4.674 ; p<0.001 ; 71-75$ years vs. $<60$ years; $S H R, 6.727,95 \% \mathrm{Cl}$, 6.049-7.482; $p<0.001 ; 76-80$ years vs. $<60$ years; $\mathrm{SHR}, 12.008,95 \% \mathrm{Cl}, 10.855-13.284 ; p<0.001 ; 81-85$ years vs. $<60$ years; SHR, $22.167,95 \% \mathrm{Cl}, 20.112-24.433 ; p<0.001 ;>85$ years vs. $<60$ years; SHR, $39.263,95 \% \mathrm{Cl}$, 35.634-43.262; $p<0.001)$. The impact of surgery was more prominent on CSM. Increasing tumor size was correlated with OS and CSM and slightly correlated with OCSM. Notably, grade and subtype differences were more predominant for CSM than for OCSM. The Kaplan-Meier curves for OS and cumulative incidence curves for CSM and OCSM were presented in Figure 2.

\section{Nomogram}

Five validated variables were incorporated to develop the prognostic nomogram: age, grade, tumor size, subtype and surgery of primary site (Figure 3). Thus, the probability of 3-, 4-, and 5-year OS, CSS and OCSS could be predicted by summing up the scores of each selected variable (higher total points, worse prognosis), helping to identify patients with high risk of cancer or non-cancer causes of death. The nomogram demonstrated considerably strong discriminative ability, with a concordance index (C-index) of 0.801 ( $95 \% \mathrm{Cl}, 0.795-0.806 ; p=0.003$ ) for the OS model (using Cox regression analysis), $0.830(95 \% \mathrm{Cl}$, 0.824-0.836; $p=0.003$ ) for the CSM and 0.806 ( $95 \% \mathrm{Cl}, 0.798-0.814 ; p=0.004)$ for the OCSM model (using Fine and Gray's competing risk analysis). Calibration plots presented high conformance between the nomogram-predicted probabilities and the observed probabilities in both the training cohort and the validation cohort (Figure 4).

The discriminatory capacity of the nomogram was evaluated by calculating the AUC values (Figure 5). The AUC values for predicting $3-, 4$-, and 5 -year OS rates were $80.2 \%, 79.5 \%$ and $78.7 \%$, respectively. As for 
the prediction of the $3-, 4$, and 5 -year CSM, the AUC values were $83.0 \%, 81.7 \%$ and $80.3 \%$, respectively. While the AUC values were $81.3 \%, 80.8 \%$ and $81.7 \%$, respectively for the $3-, 4-$, and 5 -year OCSM.

According to the C-index and AUC values, the model predicting CSM and OCSM which using Fine and Gray's competing risk analysis was more precision than that of predicting OS.

Furthermore, to further evaluate the discrimination of the model, the validation cohort was stratified into three groups by the predicted probability of the group calculated from the nomogram: low risk group, middle risk group, and high risk group. Patients of the high risk group had substantially lower OS rates and higher CSM and OCSM compared with patients of low risk group and middle risk group $(p<0.001)$ (Figure 6).

\section{Discussion}

This work analyzed the survival and mortality of early stage BC, discriminated the difference between cancer and non-cancer-related risk factors. To our knowledge, this is the first study using Fine and Gray's competing risk analysis to predict CSM and OCSM, and then build a comprehensive nomogram predicting OS, CSS and OCSS. Cox regression model analyzing variables associated with OS is just like Fine and Gray model analyzing those of CSM and OCSM. Considering the censoring is the biggest difference between the Fine and Gray model and the Cox regression model.

Age was a strong independent predictor of OS and CSM but more obvious on OCSM. That is to say, elderly patients were under the higher risk of non-cancer causes death. Consistent with our results, Chen at el. [9] revealed that elderly women exhibited worse OS but better CSS than young women, though with the absence of evaluating OCSM. These results may due to the higher frequencies of age-related comorbidities and less support of basic life care, then may resulting in high OCSM. On the contrary, due to the age-related comorbidities and poor health, insufficient treatment (non-standard radiotherapy or chemotherapy) may increase the risk of CSM. Therefore, the status of older patients remains controversial.

HR and HER2 status play an important role both in prediction and prognosis of breast cancer $[19,20]$. With the increasing use of targeted therapy, categorizing patients according to biomarkers such as $\mathrm{HR}$ and HER2, is necessary for individualized therapy [21, 22]. In our model, compared to subtype Luminal B (HR+/HER2+), triple negative (HR-/HER2-) status and HER2 enriched (HR-/HER2+) status were significant adverse factors which means breast cancer-specific survival differed by subtype, with shortest survival among HER2 enriched and triple negative subtypes. One of the reason could be recurrence. Previous studies have reported that luminal A subtype (HR+/HER2-) patients are more likely to have a better prognosis, while HER-2 enriched and triple negative subtype patients are more likely to suffer recurrence and worse outcomes [22-25]. In early stage BC, when compared to luminal A, HER-2 enriched subtype was connected with increased local recurrence; triple negative subtype was associated with both increased local and distant metastases [20]. On the other hand, high proliferative capacity as well as the lack of directed therapies (triple negative tumors do not typically express ER or overexpress HER2) may be the 
reasons why this subtypes were associated with poor clinical outcomes. So in the studies of breast cancer, it is important that subtype be evaluated in the context of other variables such as stage at diagnosis and tumor size.

In the American Joint Committee on Cancer (AJCC) staging system, except the HR and HER2 status, tumor size and grade are important predictors as well. In our study, tumor size had a great impact on OS and CSM but little impact on OCSM. There is a strong connection between tumor size and long-term survival. The smaller tumor is, the lower T stage is and generally resulting in a better prognosis [26]. Additionally, previous breast cancer screening study have also highlighted that earlier stage at diagnosis, better outcomes [27]. However, a previous meta-analyses showed that tumor size was not significantly related to an increase in OS [28]. In agreement with Rakha et al. [29], we also found that histologic grade predicted well on CSM and had little influence on OCSM. Now, tumor grade is already incorporated into the $8^{\text {th }}$ AJCC staging system, which is a recognition of its importance. Ignoring histologic grade in clinical decision making is inadvisable and may result in overtreatment [30].

Surgery, as the first-line treatment choice for early stage BC, was a strong predictor among OS, CSM and OCSM. In our study, based on those who underwent surgery, regardless of the surgery type, patients with absence of surgery had significantly poorer prognosis (OS: HR, 3.428; 95\% Cl, 3.277-3.641; $p<0.001$; CSM: SHR, 4.031; 95\% Cl, 3.687-4.406; $p<0.001$; OCSM: SHR, 2.037; 95\% Cl, 3.687-4.4061.851-2.424; $p<0.001$ ). Almost $90 \%$ of women diagnosed with breast cancer have early stage disease and may be treated with breast-conserving surgery or mastectomy [31,32]. Although the majority of early stage BC women have indications for breast-conserving surgery, radiation therapy and sometimes chemotherapy and/or endocrine therapy are required after breast-conserving surgery, which carry their own set of risks and adverse effects, leading to the increase of OCSM.

Although this study presents a well predictive nomogram, there are still several limitations. First, due to the unavailable subtype information before 2010 in the SEER database, the follow-up (2010-2016) time was short for early stage BC. Longer follow-up may improve the precision and discrimination of our model. The second limitation is the lacking variable of comorbidity. Instead, we consider age as a replacement of the comorbidity to make up for the limitation. Last, internal validation was used to evaluate the the model. Although it demonstrates a good accuracy, external validation based on other patients cohorts is still needed.

\section{Conclusions}

In conclusion, we evaluated OS and CIF of cancer-specific death and other-cause-specific death in patients with early stage BC based on Cox regression analysis and Fine and Gray's competing risk analysis. It is the first time to develop a comprehensive nomogram predicting 3-, 4-, and 5-year OS, CSS and OCSS in patients with early stage BC, using survival model and competing risk model. Additionally, the well-performed nomogram may help to answer patients' consultation and offer prognostic assessment for individuals. However, more studies are required for further external validation. In the near 
future, we decide to gather information about patients with early stage $\mathrm{BC}$ in our own center and other centers in China to perfect this model as well as compare the different prognostic factors between western countries and China, thus making treatment regimens suitable for Chinese people.

\section{Abbreviations}

BC, breast cancer; SEER, Surveillance, Epidemiology, and End Results; CIF, Cumulative incidence function; OS, overall survival; CSS, cancer-specific survival; OCSS, other-cause-specific survival; ROC, receiver operating characteristic; AUC, area under receiver operating characteristic; CSM, cancer-specific mortality; OCSM, other-cause-specific mortality; C-index, concordance index; $\mathrm{Cl}$, confidence interval; USA, the United States of America; IQRs, interquartile ranges; HR, hormone receptor; HER2, human epidermal growth factor receptor-2; SHR, subdistribution hazard ratio; ER, estrogen receptor; AJCC, American Joint Committee on Cancer.

\section{Declarations}

\section{Ethics approval and consent to participate}

Not applicable.

\section{Consent for publication}

Not applicable.

\section{Availability of data and materials}

The dataset supporting the conclusions of this article is available in the Surveillance, Epidemiology, and End Results (SEER) database, [https://seer.cancer.gov/]

\section{Competing interests}

The authors declare that they have no competing interests.

\section{Funding}

Not applicable. 


\section{Authors' contributions}

YBX and DX designed this paper and performed critical revision of the manuscript. HL, QHC, JLJ and RRD performed data collection and analyzed the data. YBX wrote the manuscript. All authors read and approved the final manuscript.

\section{Acknowledgement}

Not applicable.

\section{References}

1. Siegel RL, Miller KD. Cancer statistics, 2019. CA Cancer J Clin. 2019; 69: 7-34.

2. Perez EA, Romond EH, Suman VJ, Jeong JH, Sledge G, Geyer CE, et al. Trastuzumab plus adjuvant chemotherapy for human epidermal growth factor receptor 2-positive breast cancer: planned joint analysis of overall survival from NSABP B-31 and NCCTG N9831. J Clin Oncol. 2014; 32: 3744-52.

3. Giordano SH, Temin S, Kirshner JJ, Chandarlapaty S, Crews JR, Davidson NE, et al. Systemic therapy for patients with advanced human epidermal growth factor receptor 2-positive breast cancer: American Society of Clinical Oncology clinical practice guideline. J Clin Oncol. 2014; 32: 2078-99.

4. Harris LN, Ismaila N, McShane LM, Andre F, Collyar DE, Gonzalez-Angulo AM, et al. Use of Biomarkers to Guide Decisions on Adjuvant Systemic Therapy for Women With Early-Stage Invasive Breast Cancer: American Society of Clinical Oncology Clinical Practice Guideline. J Clin Oncol. 2016; 34: 1134-50.

5. Chen Y, Zhang Y, Yang W, Li X, Zhu L, Chen K, et al. Accuracy of a nomogram to predict the survival benefit of surgical axillary staging in T1 breast cancer patients. Medicine. 2018; 97: e11273.

6. Carroll R, Lawson AB, Jackson CL, Zhao S. Assessment of spatial variation in breast cancer-specific mortality using Louisiana SEER data. Soc Sci Med. 2017; 193: 1-7.

7. Abdel-Rahman O. Assessment of the prognostic and discriminating value of the novel bioscore system for breast cancer; a SEER database analysis. Breast Cancer Res Tr. 2017; 164: 231-6.

8. Yi M, Mittendorf EA, Cormier JN, Buchholz TA, Bilimoria K, Sahin AA, et al. Novel staging system for predicting disease-specific survival in patients with breast cancer treated with surgery as the first intervention: time to modify the current American Joint Committee on Cancer staging system. J Clin Oncol. 2011; 29: 4654-61.

9. Chen HL, Zhou MQ, Tian W, Meng KX, He HF. Effect of Age on Breast Cancer Patient Prognoses: A Population-Based Study Using the SEER 18 Database. PloS one. 2016; 11: e0165409. 
10. Zhang Z, Geskus RB, Kattan MW, Zhang H, Liu T. Nomogram for survival analysis in the presence of competing risks. Ann Transl Med. 2017; 5: 403.

11. Pintilie M. Competing risks: a practical perspective. 1st ed. Chichester, England: John Wiley \& Sons; 2006.

12. Carmona R, Zakeri K, Green G, Hwang L, Gulaya S, Xu B, et al. Improved Method to Stratify Elderly Patients With Cancer at Risk for Competing Events. J Clin Oncol. 2016; 34: 1270-7.

13. Fine JP, Gray RJ. A Proportional Hazards Model for the Subdistribution of a Competing Risk. J Am Stat Assoc. 1999; 94: 496-509.

14. Gray RJ. A class of K-sample tests for comparing the cumulative incidence of a competing risk. Ann Stat. 1988; 16: 1141-54.

15. Wang Y, Li J, Xia Y, Gong R, Wang K, Yan Z, et al. Prognostic nomogram for intrahepatic cholangiocarcinoma after partial hepatectomy. J Clin Oncol. 2013; 31: 1188-95.

16. Harrell FE, Jr., Lee KL, Mark DB. Multivariable prognostic models: issues in developing models, evaluating assumptions and adequacy, and measuring and reducing errors. Stat Med. 1996; 15: 36187.

17. Pencina MJ, D'Agostino RB. Overall $C$ as a measure of discrimination in survival analysis: model specific population value and confidence interval estimation. Stat Med. 2004; 23: 2109-23.

18. Kim Y, Margonis GA, Prescott JD, Tran TB, Postlewait LM, Maithel SK, et al. Nomograms to Predict Recurrence-Free and Overall Survival After Curative Resection of Adrenocortical Carcinoma. JAMA surgery. 2016; 151: 365-73.

19. Wang Y, Klijn JG, Zhang Y, Sieuwerts AM, Look MP, Yang F, et al. Gene-expression profiles to predict distant metastasis of lymph-node-negative primary breast cancer. Lancet. 2005; 365: 671-9.

20. Nguyen PL, Taghian AG, Katz MS, Niemierko A, Abi Raad RF, Boon WL, et al. Breast cancer subtype approximated by estrogen receptor, progesterone receptor, and HER-2 is associated with local and distant recurrence after breast-conserving therapy. J Clin Oncol. 2008; 26: 2373-8.

21. Perou CM, Sorlie T, Eisen MB, van de Rijn M, Jeffrey SS, Rees CA, et al. Molecular portraits of human breast tumours. Nature. 2000; 406: 747-52.

22. Sorlie T, Perou CM, Tibshirani R, Aas T, Geisler S, Johnsen H, et al. Gene expression patterns of breast carcinomas distinguish tumor subclasses with clinical implications. Proc Natl Acad Sci U S A. 2001; 98: $10869-74$.

23. Sorlie T, Tibshirani R, Parker J, Hastie T, Marron JS, Nobel A, et al. Repeated observation of breast tumor subtypes in independent gene expression data sets. Proc Natl Acad Sci U S A. 2003; 100: 841823.

24. Sotiriou C, Neo SY, McShane LM, Korn EL, Long PM, Jazaeri A, et al. Breast cancer classification and prognosis based on gene expression profiles from a population-based study. Proc Natl Acad Sci U S A. 2003; 100: 10393-8.

25. Carey LA, Perou CM, Livasy CA, Dressler LG, Cowan D, Conway K, et al. Race, breast cancer subtypes, and survival in the Carolina Breast Cancer Study. JAMA. 2006; 295: 2492-502. 
26. Amin MB, Greene FL, Edge SB, Compton CC, Gershenwald JE, Brookland RK, et al. The Eighth Edition AJCC Cancer Staging Manual: Continuing to build a bridge from a population-based to a more "personalized" approach to cancer staging. CA Cancer J Clin. 2017; 67: 93-9.

27. Autier P, Hery C, Haukka J, Boniol M, Byrnes G. Advanced breast cancer and breast cancer mortality in randomized controlled trials on mammography screening. J Clin Oncol. 2009; 27: 5919-23.

28. Cortazar P, Zhang L, Untch M, Mehta K, Costantino JP, Wolmark N, et al. Pathological complete response and long-term clinical benefit in breast cancer: the CTNeoBC pooled analysis. Lancet. 2014; 384: 164-72.

29. Rakha EA, El-Sayed ME, Lee AH, Elston CW, Grainge MJ, Hodi Z, et al. Prognostic significance of Nottingham histologic grade in invasive breast carcinoma. J Clin Oncol. 2008; 26: 3153-8.

30. Lundin J, Lundin M, Holli K, Kataja V, Elomaa L, Pylkkanen L, et al. Omission of histologic grading from clinical decision making may result in overuse of adjuvant therapies in breast cancer: results from a nationwide study. J Clin Oncol. 2001; 19: 28-36.

31. Kummerow KL, Du L, Penson DF, Shyr Y, Hooks MA. Nationwide trends in mastectomy for early-stage breast cancer. JAMA surgery. 2015; 150: 9-16.

32. Boughey JC, Attai DJ, Chen SL, Cody HS, Dietz JR, Feldman SM, et al. Contralateral Prophylactic Mastectomy (CPM) Consensus Statement from the American Society of Breast Surgeons: Data on CPM Outcomes and Risks. Ann Surg Oncol. 2016; 23: 3100-5.

\section{Tables}

Table 1. Patient characteristics of the training cohort and the validation cohort. 
Training Cohort Validation Cohort $P$-value

\begin{tabular}{|c|c|c|c|}
\hline \multirow[b]{2}{*}{ Age (years) } & \multirow[t]{2}{*}{ No. (\%) } & \multirow[t]{2}{*}{ No. (\%) } & \multirow[b]{2}{*}{0.702} \\
\hline & & & \\
\hline$<60$ & 83835 (42.7) & $9312(4.7)$ & \\
\hline $60-65$ & 30234 (15.4) & 3394(1.7) & \\
\hline $66-70$ & 22145 (11.3) & 2493(1.3) & \\
\hline $71-75$ & $16528(8.4)$ & 1813(0.9) & \\
\hline $76-80$ & 11461 (5.8) & $1290(0.7)$ & \\
\hline $81-85$ & 7374 (3.8) & $772(0.4)$ & \\
\hline$>85$ & 5097 (2.6) & $556(0.3)$ & \\
\hline Grade & & & 0.996 \\
\hline I & 44718 (22.8) & 4973(2.5) & \\
\hline II & 78434 (40.0) & $8716(4.4)$ & \\
\hline III-IV & $53522(27.3)$ & $5941(3.0)$ & \\
\hline Tumor size (cm) & & & 0.841 \\
\hline$<1$ & $41174(21.0)$ & 4598(2.3) & \\
\hline $1-1.9$ & 69697 (35.5) & 7687(3.9) & \\
\hline $2-2.9$ & 37264 (19.0) & 4169(2.1) & \\
\hline $3-3.9$ & $16006(8.2)$ & 1804(0.9) & \\
\hline $4-4.9$ & 7480 (3.8) & $836(0.4)$ & \\
\hline$\geq 5$ & $5053(2.6)$ & $536(0.3)$ & \\
\hline Subtype & & & 0.453 \\
\hline HR-/HER2- & 19017 (9.7) & 2183(1.1) & \\
\hline (Triple Negative) & & & \\
\hline HR-/HER2+ & $6976(3.6)$ & $760(0.4)$ & \\
\hline (HER2 Enriched) & & & \\
\hline HR+/HER2- & 132675 (67.6) & $14708(7.5)$ & \\
\hline
\end{tabular}


(Luminal A)

HR+/HER2+ $18006(9.2) \quad 1979(1.0)$

(Luminal B)

\begin{tabular}{|c|c|c|c|}
\hline Surgery to primary sites & & & 0.901 \\
\hline No & $5268(2.7)$ & $589(0.3)$ & \\
\hline Yes & $171406(87.3)$ & 19041(9.7) & \\
\hline
\end{tabular}

No., number; cm, centimeter; HR, hormone receptor; HER2, human epidermal growth factor receptor type 2.

Table 2. Overall survival rates and cumulative incidences of mortality among patients with breast cancer 


\section{Overall survival rate}

(\%)

3-y 4-

Cancer-specific mortality

(\%)

4-y

$5-\mathrm{y}$

Other-cause-specific mortality

(\%)

$3-\mathrm{y}$

4-y

$5-\mathrm{y}$

\section{Age (years)}

\begin{tabular}{lllllllllll}
$<60$ & 0.974 & 0.962 & 0.952 & 0.020 & 0.029 & 0.038 & 0.006 & 0.008 & 0.010 \\
$60-65$ & 0.970 & 0.958 & 0.946 & 0.016 & 0.024 & 0.030 & 0.014 & 0.019 & 0.024 \\
\hline $66-70$ & 0.960 & 0.944 & 0.927 & 0.018 & 0.025 & 0.031 & 0.021 & 0.031 & 0.041 \\
\hline $71-75$ & 0.946 & 0.923 & 0.898 & 0.020 & 0.028 & 0.038 & 0.034 & 0.048 & 0.065 \\
\hline $76-80$ & 0.909 & 0.870 & 0.827 & 0.032 & 0.042 & 0.055 & 0.059 & 0.088 & 0.119 \\
\hline $81-85$ & 0.839 & 0.777 & 0.708 & 0.049 & 0.064 & 0.073 & 0.112 & 0.159 & 0.219 \\
$>85$ & 0.683 & 0.582 & 0.474 & 0.091 & 0.116 & 0.135 & 0.226 & 0.302 & 0.391
\end{tabular}

\section{Grade}

$\begin{array}{llllllllll}\text { I } & 0.969 & 0.955 & 0.938 & 0.005 & 0.008 & 0.011 & 0.025 & 0.037 & 0.051 \\ \text { II } & 0.958 & 0.939 & 0.920 & 0.014 & 0.021 & 0.028 & 0.028 & 0.039 & 0.052 \\ \text { III-IV } & 0.923 & 0.897 & 0.871 & 0.052 & 0.069 & 0.085 & 0.025 & 0.034 & 0.043\end{array}$

\section{Tumor size (cm)}

\begin{tabular}{lccccccccc}
\hline$<1$ & 0.978 & 0.966 & 0.954 & 0.006 & 0.008 & 0.012 & 0.016 & 0.025 & 0.035 \\
\hline $1-1.9$ & 0.964 & 0.948 & 0.930 & 0.013 & 0.019 & 0.024 & 0.024 & 0.034 & 0.046 \\
\hline $2-2.9$ & 0.939 & 0.913 & 0.888 & 0.030 & 0.044 & 0.056 & 0.031 & 0.043 & 0.056 \\
$\geq 3$ & - & - & - & - & - & - & 0.042 & 0.055 & 0.067 \\
\hline $3-3.9$ & 0.903 & 0.868 & 0.837 & 0.057 & 0.079 & 0.099 & - & - & - \\
$\geq 4$ & 0.876 & 0.840 & 0.806 & - & - & - & - & - & - \\
\hline $4-4.9$ & - & - & - & 0.076 & 0.100 & 0.120 & - & - & - \\
$\geq 5$ & - & - & - & 0.086 & 0.105 & 0.126 & - & - & - \\
\hline
\end{tabular}

\section{Subtype}

HR-/HER2-

$\begin{array}{llll}0.892 & 0.860 & 0.834 & 0.080\end{array}$

0.102

0.120

0.028

0.038

0.046

(Triple Negative)

HR-/HER2+

$\begin{array}{lllll}0.936 & 0.915 & 0.895 & 0.041 & 0.056\end{array}$

0.068

0.022

0.029

0.037 
(HER2 Enriched)

$\begin{array}{llllllllll}\text { HR+/HER2- } & 0.958 & 0.940 & 0.920 & 0.014 & 0.022 & 0.029 & 0.027 & 0.038 & 0.051\end{array}$

(Luminal A)

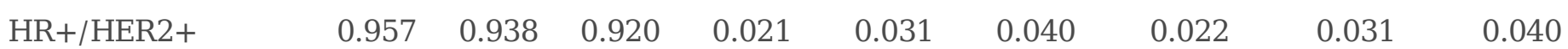

(Luminal B)

\section{Surgery to primary}

sites

\begin{tabular}{llllllllll} 
No & 0.731 & 0.664 & 0.617 & 0.146 & 0.184 & 0.211 & 0.123 & 0.152 & 0.172 \\
Yes & 0.956 & 0.937 & 0.917 & 0.020 & 0.029 & 0.037 & 0.024 & 0.034 & 0.046 \\
\hline
\end{tabular}

y, year; cm, centimeter; HR, hormone receptor; HER2, human epidermal growth factor receptor type 2.

Table 3. Outcomes of multivariate Cox analysis for OS, Fine and Gray's analysis for CSM and OCSM 


\begin{tabular}{|c|c|c|c|c|c|c|c|c|c|}
\hline & \multicolumn{3}{|c|}{ OS } & \multicolumn{3}{|c|}{ CSM } & \multicolumn{3}{|c|}{ OCSM } \\
\hline & HR & $95 \% \mathrm{CI}$ & $p$-value & SHR & $95 \%$ & $p$-value & SHR & $95 \% \mathrm{CI}$ & $p$-value \\
\hline & & & & & $\mathrm{CI}$ & & & & \\
\hline \multicolumn{10}{|c|}{ Age (years) } \\
\hline$<60$ & reference & - & - & reference & - & - & reference & - & - \\
\hline \multirow[t]{2}{*}{$60-65$} & 1.416 & $1.322-$ & $p<0.001$ & 1.082 & $0.989-$ & $p=0.084$ & 2.561 & 2.283- & $p<0.001$ \\
\hline & & 1.516 & & & 1.184 & & & 2.873 & \\
\hline \multirow[t]{2}{*}{$66-70$} & 2.105 & $1.879-$ & $p<0.001$ & 1.306 & $1.181-$ & $p<0.001$ & 4.182 & 3.742- & $p<0.001$ \\
\hline & & 2.162 & & & 1.443 & & & 4.674 & \\
\hline \multirow[t]{2}{*}{$71-75$} & 2.900 & 2.707- & $p<0.001$ & 1.545 & $1.389-$ & $p<0.001$ & 6.727 & 6.049- & $p<0.001$ \\
\hline & & 3.107 & & & 1.717 & & & 7.482 & \\
\hline \multirow[t]{2}{*}{$76-80$} & 4.914 & $4.604-$ & $p<0.001$ & 2.208 & $1.988-$ & $p<0.001$ & 12.008 & $10.855-$ & $p<0.001$ \\
\hline & & 5.245 & & & 2.453 & & & 13.284 & \\
\hline \multirow[t]{2}{*}{ 81-85 } & 8.292 & 7.878- & $p<0.001$ & 2.780 & $2.493-$ & $p<0.001$ & 22.167 & 20.112- & $p<0.001$ \\
\hline & & 8.829 & & & 3.099 & & & 24.433 & \\
\hline \multirow[t]{2}{*}{$>85$} & 14.169 & $13.340-$ & $p<0.001$ & 3.852 & $3.469-$ & $p<0.001$ & 39.263 & 35.634- & $p<0.001$ \\
\hline & & 15.050 & & & 4.276 & & & 43.262 & \\
\hline
\end{tabular}

\section{Grade}

$\begin{array}{lrrrrrr}\text { I } & \text { reference } & - & - & \text { reference } & - & - \\ \text { II } & 1.135 & 1.075- & p<0.001 & 2.084 & 1.845- & p<0.001 \\ & & 1.199 & & & 2.353 & \\ \text { III-IV } & 1.703 & 1.602- & p<0.001 & 4.486 & 3.956- & p<0.001 \\ & & 1.809 & & & 5.087 & \end{array}$

\section{Tumor size}

(cm)

$\begin{array}{lcccccccccc}<1 & \text { reference } & - & - & \text { reference } & - & - & \text { reference } & - & - \\ 1-1.9 & 1.445 & 1.355- & p<0.001 & 1.717 & 1.516- & p<0.001 & 1.343 & 1.247- & p<0.001 \\ & & 1.541 & & & & 1.944 & & & 1.447 & \\ 2-2.9 & 2.116 & 1.978- & p<0.001 & 3.041 & 2.686- & p<0.001 & 1.681 & 1.551- & p<0.001\end{array}$




\begin{tabular}{|c|c|c|c|c|c|c|c|c|c|}
\hline \multirow[b]{2}{*}{$\geq 3$} & \multicolumn{3}{|c|}{2.263} & \multicolumn{3}{|c|}{3.445} & \multicolumn{3}{|c|}{1.822} \\
\hline & - & - & - & - & - & - & 1.869 & 1.718- & $p<0.001$ \\
\hline & & & & & & & & 2.034 & \\
\hline \multirow[t]{2}{*}{ 3-3.9 } & 2.820 & 2.619- & $p<0.001$ & 4.589 & $4.030-$ & $p<0.001$ & - & - & - \\
\hline & & 3.037 & & & 5.226 & & & & \\
\hline \multirow[t]{2}{*}{$\geq 4$} & 3.307 & 3.069- & $p<0.001$ & - & - & - & - & - & - \\
\hline & & 3.565 & & & & & & & \\
\hline \multirow[t]{2}{*}{$4-4.9$} & - & - & - & 5.539 & $4.810-$ & $p<0.001$ & - & - & - \\
\hline & & & & & 6.380 & & & & \\
\hline \multirow[t]{2}{*}{$\geq 5$} & - & - & - & 5.851 & $5.032-$ & $p<0.001$ & - & - & - \\
\hline & & & & & 6.804 & & & & \\
\hline
\end{tabular}

\section{Subtype}

HR+/HER2+ reference

reference

(Luminal B)

\begin{tabular}{|c|c|c|c|c|c|}
\hline HR-/HER2- & 1.859 & $1.724-$ & $p<0.001$ & 2.460 & 2.219 \\
\hline & & 2.004 & & & 2.727 \\
\hline
\end{tabular}

(Triple

Negative)

$\begin{array}{lrrrr}\text { HR-/HER2+ } & 1.167 \quad 1.048-\quad p=0.005 \quad 1.384 & 1.200- & p<0.001 \\ & 1.300 & & 1.595\end{array}$

(HER2

Enriched)
HR+/HER2-
1.000
0.934- $\quad p=0.996$
1.065
$0.960-\quad p=0.230$
(Luminal A)
1.071
1.181

\section{Surgery to}

\section{primary sites}

\begin{tabular}{cccccccccc}
\hline Yes & reference & - & - & reference & - & - & reference & - & - \\
\hline No & 3.428 & $3.227-$ & $p<0.001$ & 4.031 & $3.687-$ & $p<0.001$ & 2.037 & $1.851-$ & $p<0.001$ \\
& & & & & & & & &
\end{tabular}


OS, overall survival; CSM, cancer-specific mortality; OCSM, other-cause-specific mortality; HR, hazard ratio; CI, confidence interval; SHR, subdistribution hazard ratio; cm, centimeter; HR+, hormone receptor positive; HER2+, human epidermal growth factor receptor type 2 positive; HR-, hormone receptor negative; HER2-, human epidermal growth factor receptor type 2 negative.

\section{Figures}




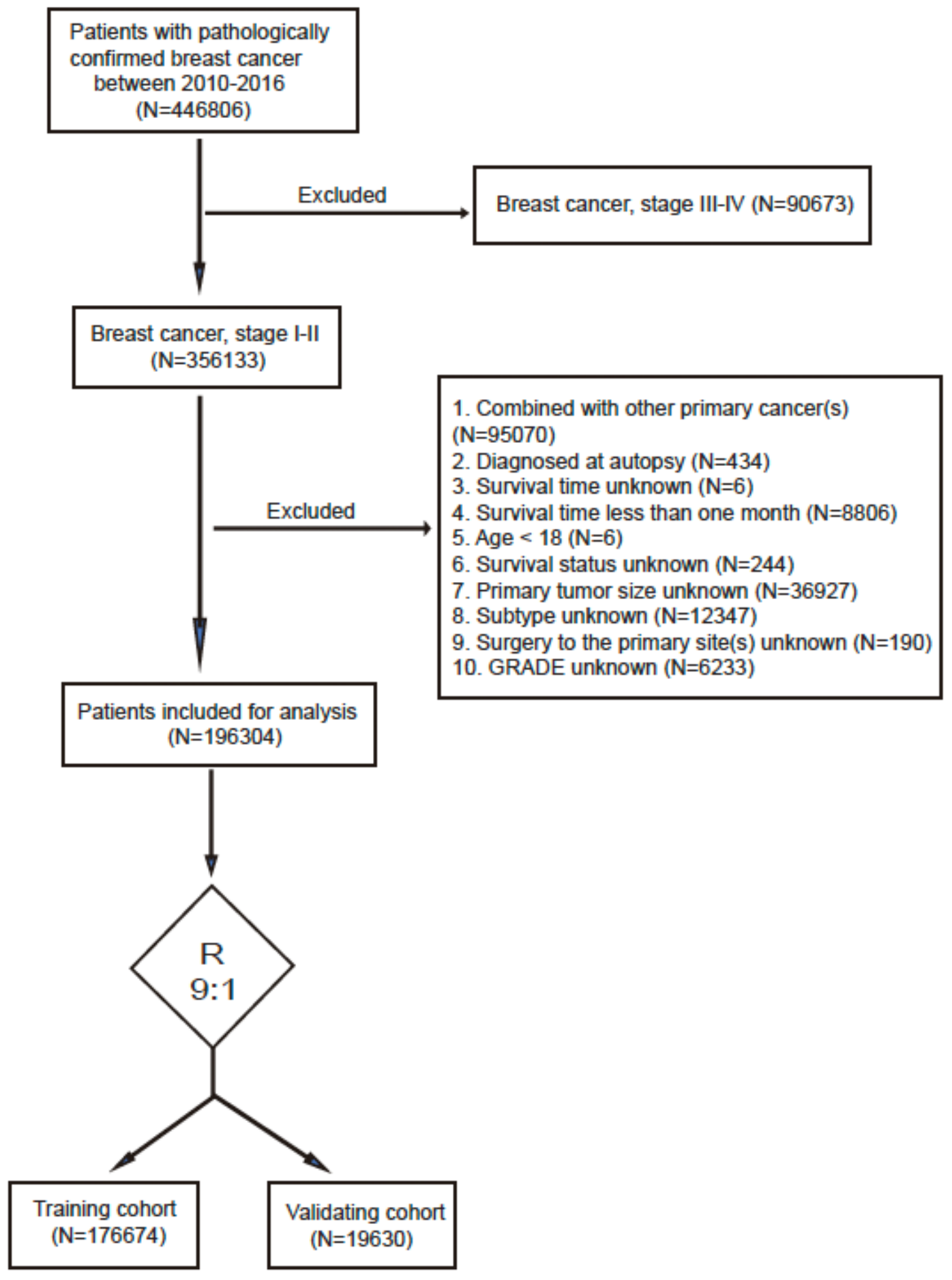

\section{Figure 1}

Flowchart of patient selection 

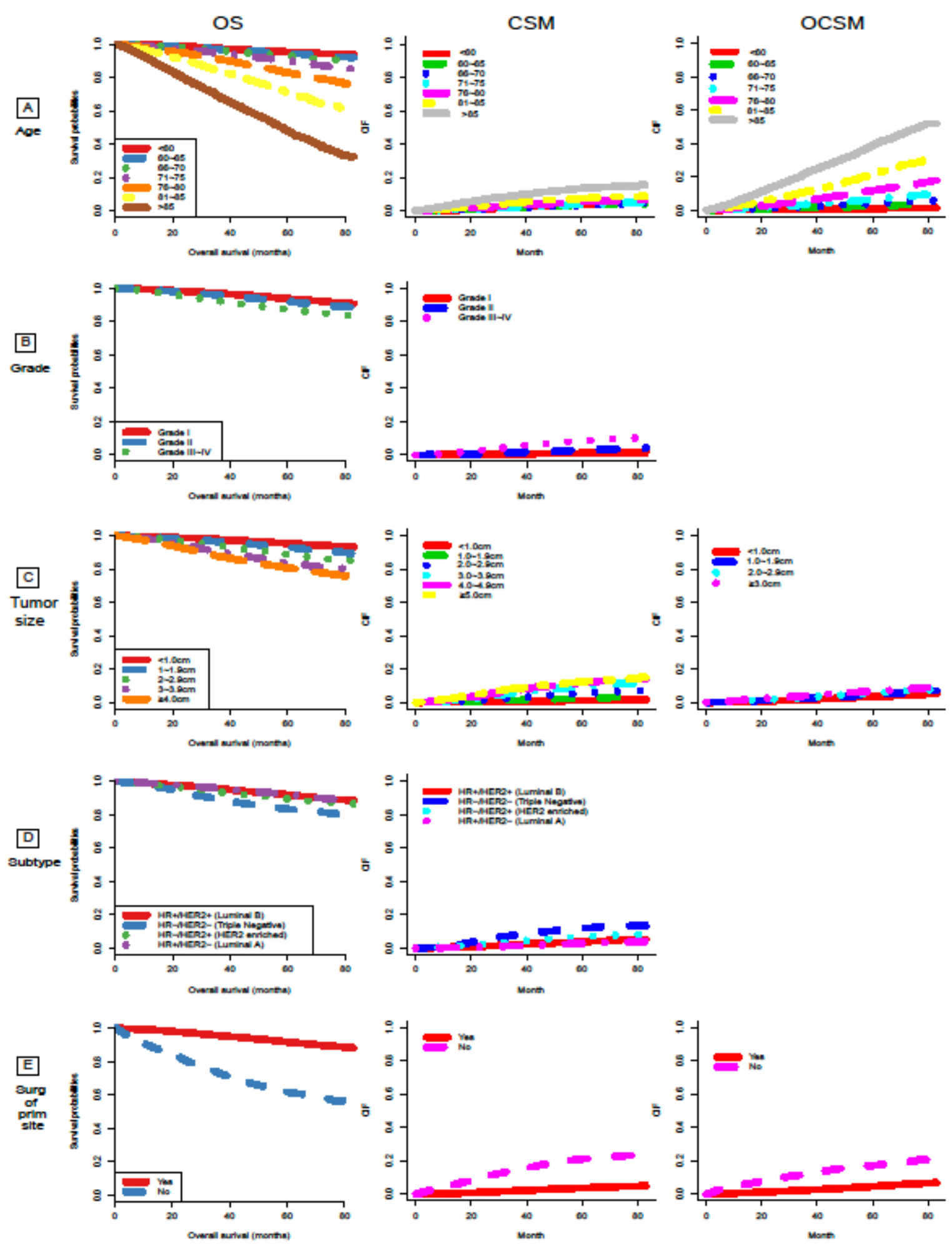

Figure 2

OS and CIF for cancer-specific death and other-cause-specific death OS and CIF for cancer-specific death and other-cause-specific death according to patient characteristics (A) age; (B) Grade; (C) Tumor size; (D) Subtype; (E) Surgery. OS, overall survival; CSM, cancer-specific mortality; OCSM, other-cause-specific mortality; $\mathrm{CIF}$, cumulative incidence function; $\mathrm{HR}+$, hormone receptor positive; $\mathrm{HER} 2+$, human epidermal 
growth factor receptor type 2 positive; HR-, hormone receptor negative; HER2-, human epidermal growth factor receptor type 2 negative; Surg of prim site, surgery of primary site.

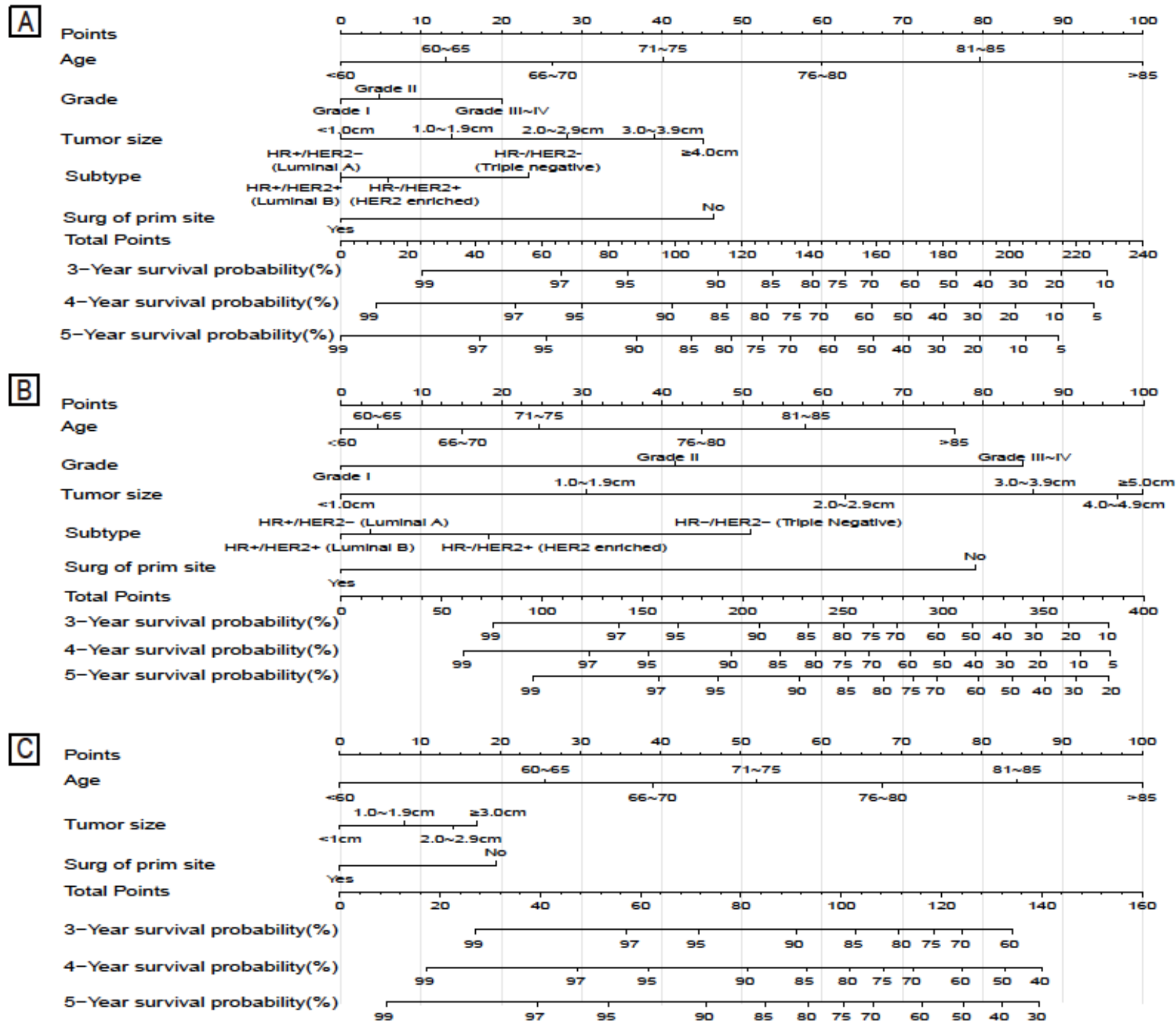

\section{Figure 3}

Nomograms predicting 3-, 4- and 5-year OS (A), CSS (B) and OCSS (C) OS, overall survival; CSS, cancerspecific survival; OCSS, other-cause-specific survival; HR+, hormone receptor positive; HER2+, human epidermal growth factor receptor type 2 positive; HR-, hormone receptor negative; HER2-, human epidermal growth factor receptor type 2 negative; Surg of prim site, surgery of primary site. OS and CIF for cancerspecific death and other-cause-specific death OS and CIF for cancer-specific death and other-causespecific death according to patient characteristics (A) age; (B) Grade; (C) Tumor size; (D) Subtype; (E) Surgery. OS, overall survival; CSM, cancer-specific mortality; OCSM, other-cause-specific mortality; CIF, cumulative incidence function; HR+, hormone receptor positive; HER2+, human epidermal growth factor 
receptor type 2 positive; HR-, hormone receptor negative; HER2-, human epidermal growth factor receptor type 2 negative; Surg of prim site, surgery of primary site.
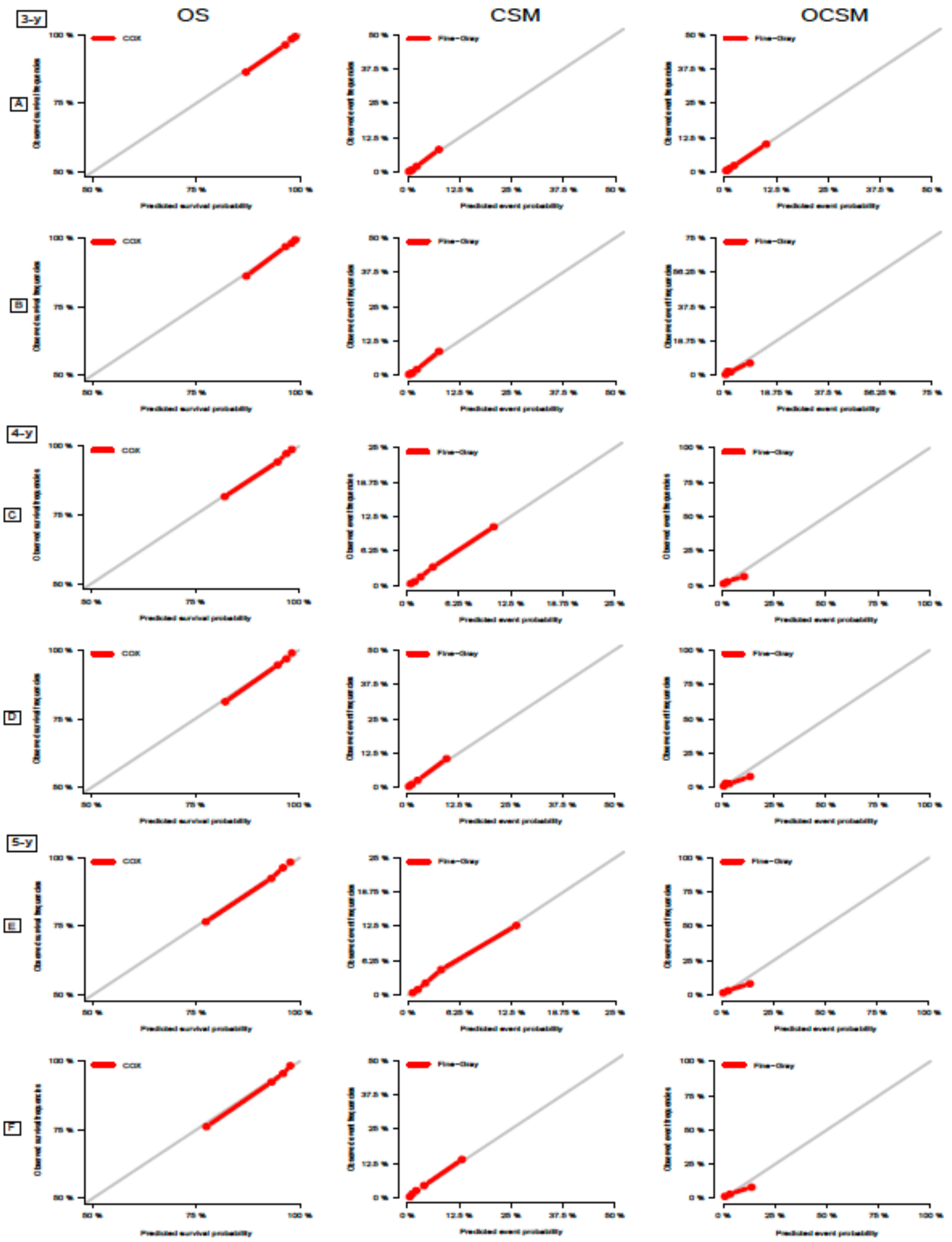

Figure 4

Calibration plots of the nomograms for 3-, 4- and 5-year OS, CSM and OCSM (A, C, E). Calibration plots of the training cohort; $(B, D, F)$. Calibration plots of validation cohort. X-axis represents the nomogram- 
predicted event probabilities; Y-axis represents the observed event frequencies. OS, overall survival; CSM, cancer-specific mortality; OCSM, other-cause-specific mortality; 3-y, 3-year; 4-y, 4-year; 5-y, 5-year.
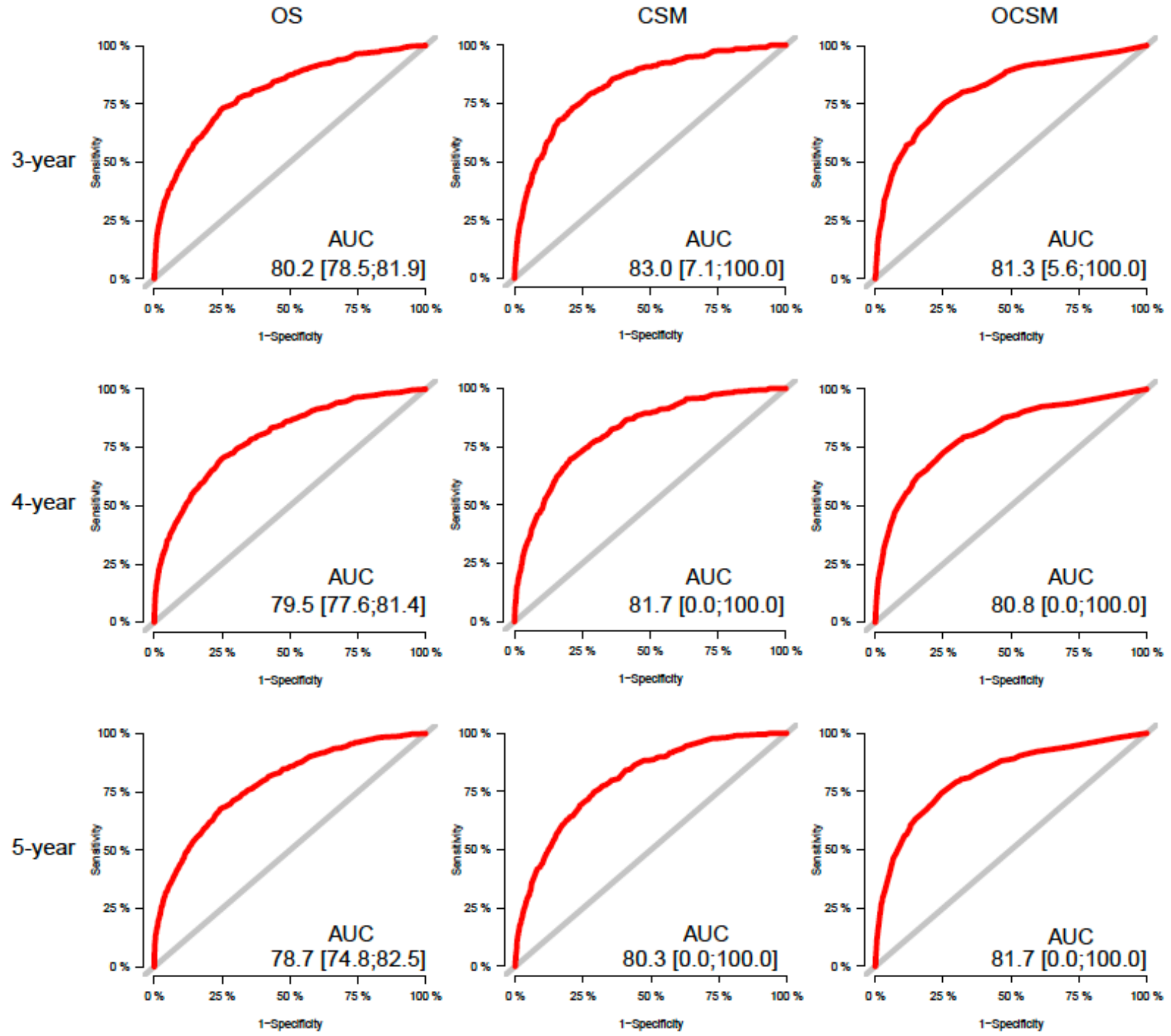

Figure 5

ROC curves for 3-, 4- and 5-year OS prediction, CSM prediction and OCSM prediction OS, overall survival; CSM, cancer-specific mortality; OCSM, other-cause-specific mortality; ROC, receiver operating characteristic curve; AUC, area under ROC. 


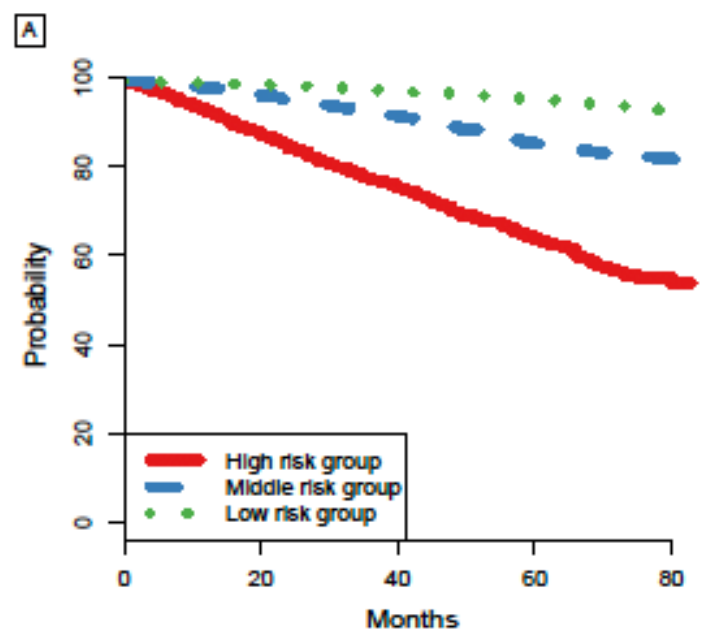

B

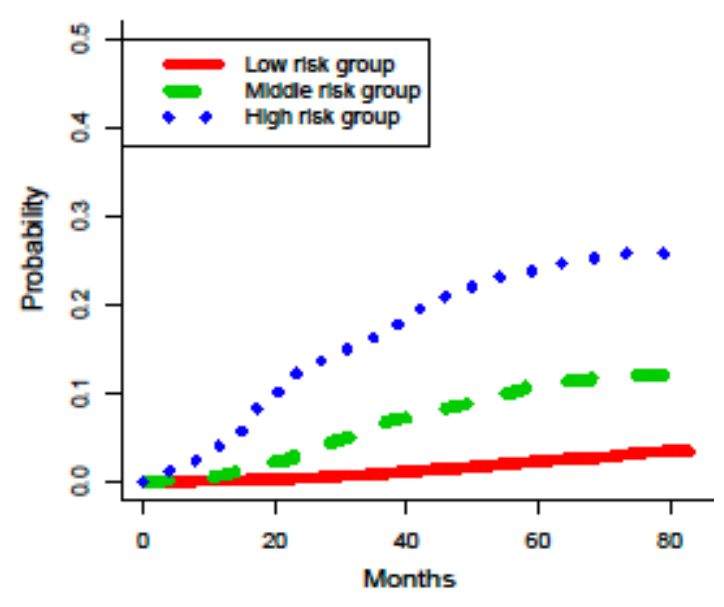

c.

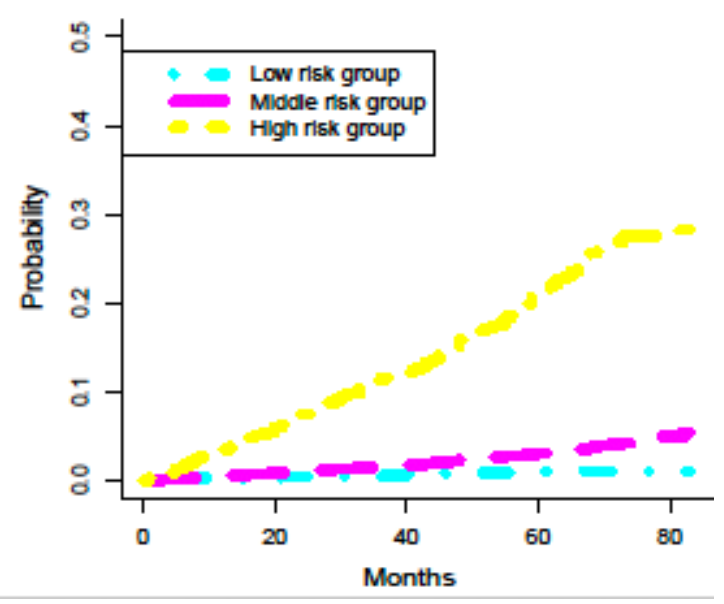

Figure 6

Kaplan-Meier and CIF curves stratified by the risk levels of nomogram-predicted probabilities (A). OS rates ; (B). CSM; C. OCSM OS, overall survival; CSM, cancer-specific mortality; OCSM, other-cause-specific mortality. 